\title{
Risk and Management of Ocular Bleeding Associated with Oral Anticoagulants
}

\author{
Len V. Koh, \\ OD, PhD, FAAO \\ Staff Optometrist \\ Mann-Granstaff VA \\ Medical Center \\ Chad E. Gosnell, \\ OD, FAAO \\ Chief of Optometry Service
}

\author{
Anna R. Well, \\ OD, FAAO \\ Staff Optometrist
}

\begin{abstract}
Deep vein thrombosis, pulmonary embolism, ischemic stroke and myocardial infarction are major thromboembolic diseases that affect millions of North Americans. Most of these patients are managed long term with oral anticoagulants that can increase the risk of bleeding, including ocular hemorrhage. Interruption of anticoagulation temporarily increases the thromboembolic risk, and continuing anticoagulation increases the risk of bleeding; both scenarios adversely affect the patient's overall health. This article provides an overview and update of oral anticoagulants, and discusses ways for optometrists to manage ocular bleeding associated with oral anticoagulants.
\end{abstract}

\section{INTRODUCTION}

Thrombosis is the formation of clots that impede blood flow resulting in tissue ischemia or damage. It can arise in both arterial and venous vessels. A thrombus can break off and move as a thromboembolus or embolus that travels through the blood vessels until it reaches a vessel that is too small to pass through, which blocks blood flow at another location in the body, including the eyes, where it can manifest as retinal embolus (Hollenhorst plaque) or retinal artery occlusion., ${ }^{1,2}$ The major thromboembolic diseases are deep vein thrombosis (DVT), pulmonary embolus (PE), ischemic stroke and myocardial infarction (MI). DVT is caused by a blood clot in a vein deep inside the body. Large veins in the lower leg and thigh, the pelvis and arm are most susceptible. While DVT per se could be benign, an embolus from DVT can move through the blood vessels and ultimately block blood perfusion to the lungs, heart, brain, or other area, leading to tissue damage, debility or death. PE is blockage of an artery in the lungs that is commonly caused by an embolus from DVT. Less common causes of PE include air bubbles, fat droplets and tumor cells. An ischemic stroke occurs when a thrombus or embolus blocks a blood vessel in the brain. Cerebral thrombosis develops via atherosclerosis, whereas cerebral embolism usually derives from a thrombus in the heart or large arteries in the upper chest and neck. Furthermore, atrial fibrillation increases the propensity for emboli to travel and block the brain circulation, leading to stroke. MI (heart attack) happens when blood flow from a coronary artery to the heart is suddenly blocked, causing cardiac muscle cell death. An insidious atherosclerotic plaque in the coronary artery poses the highest risk for a heart attack because the plaque can grow and block blood flow, or a tear in the plaque can trigger a blood clot and block the supply to the heart tissue. Collectively, these thromboembolic diseases account for up to one-fourth of all deaths worldwide. ${ }^{1}$ In 2015, 28.4 million adult Americans were diagnosed with heart disease and 6.5 million had ever had a stroke. Accordingly, heart disease and stroke are two of the leading causes of death in America, ranking first (614,348 deaths per year) and fifth $(133,103)$ respectively. 
The incidence of thrombosis is increasing as the population ages. ${ }^{3}$ Anticoagulant agents are the drugs of choice to prevent and treat potentially life-threatening thromboembolic events. However, patients on these medications are at an increased risk of bleeding, including ocular hemorrhage. Furthermore, some surgical procedures require patients to bridge or discontinue anticoagulation during the perioperative period. This article provides a brief overview and an update on oral anticoagulants, and discusses ways to manage ocular bleeding associated with oral anticoagulants.

Eye care providers often encounters patients who are taking oral anticoagulants. Some of these patients may present at the clinic with ocular bleeding, whereas others may be referred for ocular surgery. We must be informed about these new direct oral anticoagulants (DOACs) and know how to manage both scenarios. Let us look at the current state of knowledge on the risk of ocular bleeding in both clinical and surgical settings to be better prepared to care for these patients. The following two clinical cases are presented to illustrate possible encounters and management.

\section{CASE REPORTS}

\section{Case 1: rivaroxaban (Xarelto)}

A 70-year-old white male presented at the eye clinic on May $12^{\text {th }}$ with a complaint of bleeding in his right eye for about 10 days. While his eye looked to be in poor condition, he had no pain or irritation. This was the first occurrence and he had not been straining or lifting something heavy before the incident. His last comprehensive eye exam was two months previously. He was taking furosemide and metoprolol for hypertension, albuterol-ipratropium for chronic obstructive pulmonary disorder (COPD), and fleicainide and rivaroxaban for atrial fibrillation. His recent blood pressure and body mass index (BMI) were 168/91 and 33.4, respectively.

His habitual visual acuity was 20/25+ OD and 20/20- OS with refractive errors of -0.75-1.00x085 OD and -1.25$1.25 \mathrm{x} 080 \mathrm{OS}$. His pupils were equal and reactive to light, without afferent pupillary defect; extraocular movement was full without restriction; confrontation field was full to finger-counting. Cover test was orthophoric. Goldmann applanation tonometry was 18 mmHg OD, 17 mmHg OS @ 1328. Slit lamp exam revealed grade 1+ nuclear sclerotic cataract OU and subconjunctival hemorrhage OD (Figure 1). Dilated fundus exam was unremarkable with $\mathrm{C} / \mathrm{D}$ ratios of 0.30 round $\mathrm{OU}$.

Figure 1: Subconjunctival hemorrhage $O D$

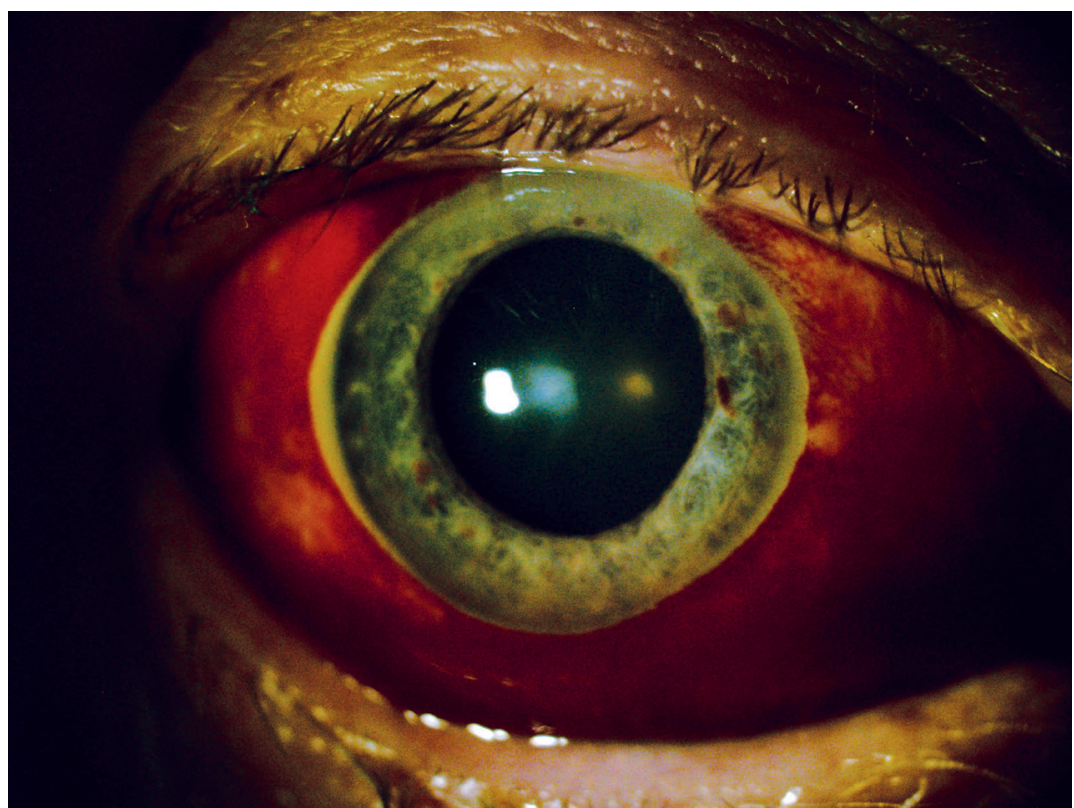


The patient was educated about the risk of bleeding while he was taking rivaroxaban. He was given artificial tears for lubrication, and told to apply a cool compress q.i.d. and to avoid straining or heavy lifting. He was reassured that the bleeding would resolve over the next few weeks and that he should return to the clinic after a few weeks unless the bleeding and vision get worse, in which case he should return sooner. His primary care provider was informed about the ocular bleeding. His latest anticoagulation work-up on Feb 16, 2016 showed an INR of 1.9 (ref. 0.8-1.14) and Protime of 21.3 (ref 11.8-13.6). The subconjunctival hemorrhage OD resolved over the next few weeks and the patient was told to continue supportive treatment and return to the clinic in one year for a complete eye examination.

\section{Case 2: warfarin (Coumadin)}

A 77-year-old white male presented at the clinic on Oct $26^{\text {th }}$ and reported slow-onset constant distance and near blur with habitual bifocals. He denied the presence of flashes, floaters, loss of vision, and ocular pain. His last eye exam was the previous year. He was taking citalopram for depression, ranitidine for gastroesophageal reflux disease, carvedilol and spironolactone for hypertension, and warfarin for aortic valve insufficiency and chronic atrial fibrillation. His last BP and BMI were 127/86 and 27, respectively.

His habitual visual acuity was 20/40-2 PH20/30-2 OD and 20/30-2 PHNI OS with refractive errors of $+0.75-1.00 x 070$ OD and +1.75-1.00x105 OS. His pupils were equal and reactive to light without afferent pupillary defect; extraocular movement was full without restriction; confrontation field was full to finger-counting. Cover test was orthophoric. Goldmann applanation tonometry was $15 \mathrm{mmHg}$ OD, 16 mmHg OS @ 0730. Slit lamp exam revealed grade 2+ mixed cataract OU. Dilated fundus exam revealed C/D ratios of 0.20 OD and 0.25 OS. Peripheral retina showed a large area of choroidal hemorrhage with curvilinear ring exudates OS (Figure 2).

Figure 2: Choroidal hemorrhage with curvilinear ring exudates OS

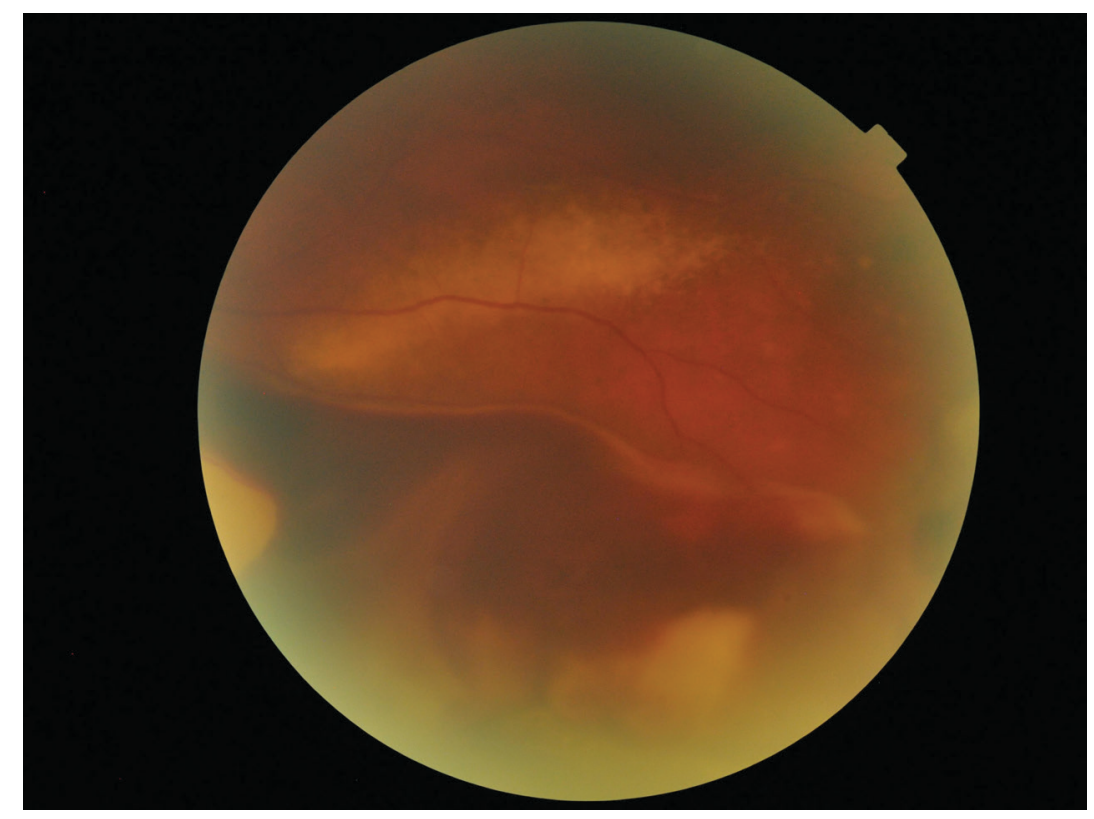

Patient was referred to a local retinal specialist for further examination and management. He was diagnosed with peripheral exudative hemorrhagic chorioretinopathy (PEHCR) and continued to be managed with anti-VEGF injections by the retinal specialist. His anticoagulation team was notified about the ocular finding. However, the retinal specialist did not recommend the discontinuation of oral anticoagulant; i.e., this may have been only a coincidental association and not a cause of PEHCR. The patient was recommended to return for routine eye care after the completion of retinal disorder management by the retinal specialist. Review of the patient's record confirmed that the patient was taking half a tablet of warfarin $(2.5 \mathrm{mg})$ by mouth at bedtime, except that one full tablet was taken on Sunday, Tuesday and Thursday. The results of recent anticoagulation tests are shown in Table 1. 
Table 1: Regular INR and PT monitoring for a patient who was taking warfarin (Case 2)

\begin{tabular}{|l|l|l|}
\hline DATE & INR & PT \\
\hline MAY 16 & 2.3 (ref. 0.8-1.14) & 25.4 (ref. 12-14.9) \\
\hline JUN 21 & 2.3 & 25.8 \\
\hline JUN 30 & 2.2 & 25.2 \\
\hline JUL 26 & 2.4 & 26.2 \\
\hline AUG 23 & 2.6 & 28.1 \\
\hline SEP 27 & 1.6 & 19.5 \\
\hline OCT 13 & 2.5 & 27.6 \\
\hline NOV 14 & 1.8 & 21.3 \\
\hline
\end{tabular}

INR, international normalized ratio; PT, prothrombin time

\section{DISCUSSION}

\section{Clotting process}

Proteases, cofactors, and protease inhibitors in the plasma participate in complex, sequentially regulated interactions to generate fibrin, which stabilizes a platelet plug or thrombus. This thrombus is the final product of the blood coagulation process and is made up of activated platelets plus a fibrous protein, fibrin (Factor Ia). ${ }^{4}$ Briefly, the blood coagulation cascade can be started by an intrinsic or extrinsic pathway. The intrinsic pathway is activated by internal damage to the blood vessel, exposing collagen to circulating platelets. It is slower than the extrinsic pathway and involves Factors XII, XI, IX and VIII. The extrinsic pathway is activated by external trauma that causes blood to escape from the vascular system and involves Factors III and VII. Both pathways converge into the common pathway by activating Factor X. In the common pathway, prothrombin (Factor II) is activated to thrombin (Factor IIa) and fibrinogen (Factor I) is activated to fibrin, which glues the platelets with the help of Factor XIII to stabilize the clot (Figure 3). ${ }^{5}$ This understanding of the clotting process provides a good foundation to grasp the mechanisms of action of oral anticoagulants.

Figure 3: Coagulation cascade and oral anticoagulant targets ${ }^{4}$

FXa indicates factorXa; HK, high-molecular weight kininogen; PK, prekallefrein; PL, phospholipid; and TF, tissue Factor

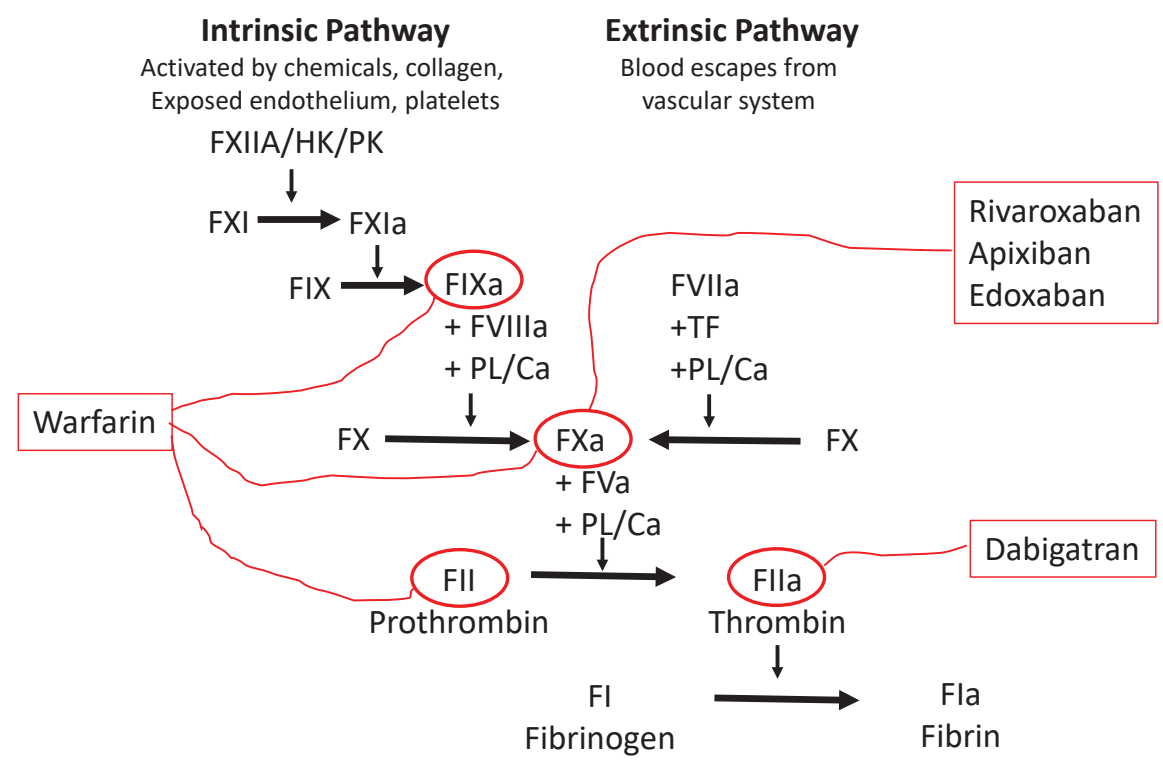




\section{Classes of anticoagulants}

Blood coagulation or clot formation depends on interactions between multiple clotting factors and platelets. This complex cascade opens up various therapeutic targets to regulate and mitigate thrombosis. Currently, three main classes of antithrombotic drug are available to manage blot clots. Anticoagulant drugs include unfractionated heparin, low molecular weight heparin (LMWH), warfarin, and direct oral anticoagulants (DOACs). They are administered subcutaneously and usually, for patients who are at risk of thrombus, in the slow-flowing blood of the veins. ${ }^{6}$ Oral antiplatelet drugs include aspirin, clopidogrel (Plavix) and dipyridamole (Persatine). They are prescribed most often for patients with a risk of clots from the fast-flowing arterial system. Aspirin irreversibly blocks the enzyme cyclooxygenase and reduces the production of thromboxane A2, which is a potent stimulator of platelet aggregation. Clopidogrel irreversibly blocks the P2Y12 component of ADP receptors on the platelet surface, which prevents activation of the GPIIb/IIa receptor complex, thereby reducing platelet aggregation. Dipyridamole inhibits the activities of adenosine deaminase and phosphodiesterase, which causes the accumulation of antiplatelet mediators such as adenosine, adenine nucleotides, and cyclic AMP. ${ }^{7}$ Thrombolytic or fibrinolytic drugs include alteplase (TPA), reteplase (Retavase) and streptokinase (Streptase). They are administered intravenously in the emergency room to break pre-existing clots within the lung, heart or brain. Alteplase initiates local fibrinolysis by binding to fibrin in a thrombus and converts entrapped plasminogen to plasmin, which degrades fibrin. Reteplase is a recombinant plasminogen activator that has a longer half-life than alteplase. Streptokinase is a single-chain polypeptide derived from beta-hemolytic streptococcus cultures. It binds to plasminogen and converts it into active plasmin to degrade fibrin clots. This article focuses mainly on oral anticoagulants including DOACs, but also mentions other classes of antithrombotic drugs.

\section{Vitamin K Antagonists (VKAs)}

Warfarin (Coumadin), the most commonly prescribed VKA, was approved in 1955 for the treatment of thromboembolic complications associated with atrial fibrillation. It is still commonly associated with rat poison because it was originally formulated with a corn base as an effective rat poison in farms. President Dwight D. Eisenhower was among the first patients to be given warfarin when he had a heart attack while on vacation in 1955. Warfarin remained the mainstay therapy for long-term anticoagulation and prevention of various thromboembolic diseases for over half a century. Coumarins, including acenocoumarol, phenprocoumon, and fluindione, are other VKAs; they are rarely used in the U.S. and hence are only mentioned in passing. The clinical challenges with warfarin are its narrow therapeutic range and various drug interactions. It is difficult and time-consuming to keep patients in the safe therapeutic range, which requires balancing the risk of thrombosis on one hand with bleeding on the other hand. The advantages of warfarin are that its use is supported by a large body of clinical data, it is inexpensive, and there are various ways to reverse its anticoagulant effects, such as with vitamin K, fresh frozen plasma (FFP) and prothrombin complex concentrates (PCCs). Necessary baseline tests prior to starting warfarin include prothrombin time (PT) with an international normalized ratio (INR) and activated partial thromboplastin time (aPTT); complete blood count, serum creatinine, liver function tests, and a pregnancy test for women of childbearing age. ${ }^{8}$

INR was proposed in 1985 as a standard measure for monitoring warfarin therapy by eliminating interlaboratory differences in test results caused by the use of thromboplastins with different sensitivities. The INR is calculated by raising the prothrombin time ratio (PTR: the patient's prothrombin time divided by a reference normal prothrombin time) to the power of a coefficient known as the international sensitivity index (ISI). ${ }^{9}$ The recommended initial daily dose is $5 \mathrm{mg}$ for the first two days, and dosing on day 3 and subsequent days is based on the PT/INR. The maintenance dose ranges from $<2 \mathrm{mg}$ to $\geq 10 \mathrm{mg}$ per day. Typical INR goals are in the range of 2.0 to 3.0 in patients with venous thromboembolism, and somewhat higher in patients with mechanical heart valves. The monitoring interval ranges from daily in hospitalized patients to up to 12 weeks in the most stable patients. Patients on warfarin must be warned to return to the clinic when a bleeding complication is experienced, such as unexplained bruising, nose bleeds and blood in the urine or stool. Furthermore, certain foods and medications can increase or decrease the efficacy of warfarin. For example, spinach, kale, and collard greens contain high levels of vitamin $\mathrm{K}$ and may render warfarin less effective..$^{10} \mathrm{~A}$ clinical pearl here is to be careful when educating age-related macular degeneration (AMD) patients who are taking warfarin to eat more green leafy vegetables, since this may affect the efficacy of anticoagulation and increase the risk of thrombosis. 


\section{Direct Oral AntiCoagulants (DOACs)}

Over the past decade, a new wave of novel oral anticoagulants has arrived to overcome warfarin's need for variable dosing and regular monitoring. This class of drugs has also been referred to as target-specific oral anticoagulants (TSOACs), oral direct inhibitors (ODIs), novel oral anticoagulants (NOACs), and non-vitamin K antagonist oral anticoagulants. ${ }^{11}$ DOACs can be classified as direct thrombin (Factor IIa) inhibitors (DTIs): dabigatran (Pradaxa), or direct Factor Xa inhibitors: rivaroxaban (Xarelto), apixaban (Eliquis), edoxaban (Savaysa), and betrixaban (Bevyxxa). Note that the generic names for these latter agents all end in "Xa-ban". These new agents permit fixed dosing and infrequent monitoring, and have become popular substitutes for warfarin. Nonetheless, warfarin is still widely used because these new DOACs lack an effective antidote and clinical experience, and have a higher cost. ${ }^{12}$ Overall, clinicians now have a much larger armamentarium for the chronic management of thromboembolic diseases. Generally, laboratory testing such as platelet count, prothrombin time (PT), and activated partial thromboplastin time (aPTT) are necessary to assess and document the coagulation status before anticoagulation with either warfarin or DOACs. Measurement of serum creatinine and liver function tests are also performed for dose-adjustment in the event of renal or hepatic insufficiency. Fortuitously, routine laboratory monitoring of coagulation time is not required for DOACs, unless bleeding or suspected overdose is evident.

Dabigatran etexilate (Pradaxa, 220-300 mg/d), the only oral DTI, is an orally administered prodrug that is converted in the liver to dabigatran, which inhibits both clot-bound and circulating thrombin. It is prone to breakdown from moisture, and hence is packaged with a desiccant. The pills must be consumed within four months to assure optimal potency. Dabigatran is used for the prevention and management of VTE disease, and to prevent stroke in patients with atrial fibrillation. It should not be used in patients with prosthetic heart valves or during pregnancy. Recently, idarucizumab (Praxbind) was approved to reverse the anticoagulant effect of dabigatran in life-threating bleeding. It is administered intravenously as 2 separate $2.5 \mathrm{~g}$ doses, no more than 15 minutes apart. ${ }^{12}$

Rivaroxaban (Xarelto, 10-30 mg/d), apixaban (Eliquis, 5-10 mg/d), edoxaban (Savaysa, 30-60 mg/d), and betrixaban (Bevyxxa, 80-160 mg/d) are direct Factor Xa inhibitors that prevent the conversion of prothrombin to thrombin. They bind directly to Factor Xa rather than enhancing the activity of antithrombin as mediated by heparin. They are indicated for the prevention and treatment of VTE disease and to prevent stroke in patients with atrial fibrillation. The dosing for this group of drugs varies depending on the clinical indication and the patient's renal function. Rivaroxaban is the only drug in this class that needs to be taken with food for optimal efficacy. There is no specific antidote for these direct Factor Xa inhibitors, so general reversal methods are used in the hospital for life-threatening or major bleeding via the administration of an antifibrinolytic agent (tranexamic acid, epsilon-aminocaproic acid) and/or oral activated charcoal to remove unabsorbed drugs from the gastrointestinal tract, and the discontinuation of all anticoagulants (Table 2).12

Table 2: Advantages and disadvantages of DOACs versus warfarin ${ }^{8}$

\begin{tabular}{|l|l|l|}
\hline & Warfarin & $\begin{array}{l}\text { DOACs } \\
\text { (dabigatran, rivaroxaban, apixaban, edoxaban) }\end{array}$ \\
\hline Dosing & Once-daily & May require more frequent dosing \\
\hline Dietary restrictions & Monitor vitamin K intake & Take rivaroxaban with food \\
\hline Monitoring & Regular PT/INR monitoring & Not required \\
\hline Drug interactions & Many & CYP-34A inhibitors and p-glycoprotein modulators \\
\hline Time in therapeutic range & $\sim 65 \%$ & $>65 \%$ \\
\hline Reversal agents & Vitamin K, FFP, PCC, rFVIIa & $\begin{array}{l}\text { Idarucizumab reverses dabigatran. Activated charcoal; } \\
\text { threatening bleeding with others. }\end{array}$ \\
\hline
\end{tabular}

DOAC, direct oral anticoagulant; PT, prothrombin time; INR, international normalized ratio; FFP, fresh frozen plasma; PCC, prothrombin complex concentrates; rFVIIa, recombinant activated Factor VII 


\section{Ocular Side Effects of DOACs}

Recently, many clinicians have turned to DOACs as the drugs of choice for the prophylaxis and treatment of thromboembolic diseases. Numerous studies have been performed to evaluate their propensity to cause major bleeding compared to warfarin, and the cumulative evidence from multiple clinical trials and other post-marketing studies has demonstrated that DOACs show equal or superior antithrombotic efficacy and lower risk of intracranial hemorrhage compared with warfarin. ${ }^{13}$ Less is known about the risk of intraocular bleeding with DOACs compared with warfarin. However, a recent meta-analysis of 12 trials investigating 102,627 patients provided some evidence-based data for eye care providers. DOACs were found to reduce the risk of intraocular bleeding by approximately $22 \%$ compared with warfarin in patients with either atrial fibrillation or venous thromboembolism. ${ }^{14}$

While the exact mechanism for the reduced risk of intraocular bleeding with DOACs compared with warfarin is unclear, they probably are safer because they target only a single site in the coagulation cascade rather than multiple sites, as with warfarin. ${ }^{13}$ This finding is particularly significant in patients with a high baseline risk of ocular bleeding, as in exudative age-related macular degeneration (AMD) and other choroidal neovascularization. Patients with AMD are 10 times more likely to have massive intraocular bleeding and a worse visual prognosis if they are taking oral anticoagulants, ${ }^{15}$ as illustrated above in Case 2.

The benefits of anticoagulants must be balanced with the risk of excess bleeding, especially in perioperative settings. It is estimated that about $10 \%$ of patients receiving anticoagulants must interrupt this treatment for surgical procedures. ${ }^{16}$ It is important for eye care providers to know whether anticoagulation needs to be interrupted prior to referring patients for ophthalmic procedures. Hemorrhagic complications in anticoagulated patients undergoing ophthalmic surgery include bloody tears, hyphema, and vitreal, subconjunctival, subretinal, and choroidal hemorrhage. ${ }^{17}$ Fortunately, the risk of severe sight-threatening hemorrhage for routine ophthalmic operations in anticoagulated patients is relatively low, and therefore the cessation of oral anticoagulants is not recommended. ${ }^{18}$ In the Randomized Evaluation of Long-term Anticoagulation therapy (RE-LY) study, no significant bleeding was found in patients taking warfarin or dabigatran who underwent cataract surgery. ${ }^{19}$ Further, no increased risk of perioperative complications was found in a small study of 36 anticoagulated patients who underwent vitreoretinal surgery. On the contrary, high-risk oculoplastic procedures, such as dacryocystorhinostomy and deep orbital and extensive eyelid surgery, require the cessation of DOACs 48 hours before surgery. ${ }^{20}$ Although there are no current guidelines on whether DOACs should be stopped prior to ophthalmic surgery, the consensus is that DOACs can be safely stopped transiently in patients with low to medium thrombotic risk, without the need for bridging therapy, whereas for higher-risk patients, the risk of thromboembolism needs to be weighed against the usually low risk of sightthreatening ocular hemorrhage (Table 3$){ }^{21}$

Table 3: Pre- and post-operative management of patients taking DOACs ${ }^{8,17}$

\begin{tabular}{|l|l|l|l|l|}
\hline Drug & MOA & Half-life, frequency & Minor Surgery & Major Surgery \\
\hline Apixaban & Factor Xa inhibitor & 12 h, b.i.d. & $\begin{array}{l}\text { Stop 2 days before } \\
\text { Restart 1 day after }\end{array}$ & $\begin{array}{l}\text { Stop 3 days before } \\
\text { Restart 2 days after }\end{array}$ \\
\hline $\begin{array}{l}\text { Dabigatran } \\
\text { prodrug) }\end{array}$ & $\begin{array}{l}\text { Direct thrombin } \\
\text { Factor IIa) } \\
\text { inhibitor }\end{array}$ & 12-17 h, b.i.d. & $\begin{array}{l}\text { Stop 2 days before } \\
\text { Restart 1 day after }\end{array}$ & $\begin{array}{l}\text { Stop 3 days before } \\
\text { Restart 2 days after }\end{array}$ \\
\hline $\begin{array}{l}\text { Edoxaban } \\
\text { (take with food) }\end{array}$ & Factor Xa inhibitor & 9-11 h, q.i.d. & $\begin{array}{l}\text { Stop 2 days before } \\
\text { Restart 1 day after }\end{array}$ & $\begin{array}{l}\text { Stop 3 days before } \\
\text { Restart 2 days after }\end{array}$ \\
\hline Warfarin & $\begin{array}{l}\text { Vitamin } \\
\text { K-dependent } \\
\text { factor synthesis }\end{array}$ & 36-42 h, q.i.d. & $\begin{array}{l}\text { Stop 2 days before } \\
\text { Restart 1 day after }\end{array}$ & $\begin{array}{l}\text { Stop 3 days before } \\
\text { Restart 2 days after }\end{array}$ \\
\hline
\end{tabular}

DOAC, direct oral anticoagulant; MOA, mechanism of action; INR, international normalized ratio 


\section{Patient Education}

Anticoagulant medicines are used to prevent blood clots seen with atrial fibrillation (which can cause blood to pool in the upper chambers of the heart), heart valve replacement (where blood clots may form on or near the heart valve), left ventricular dysfunction or cardiomyopathy (which can cause blood to pool in the lower chambers of the heart), deep vein thrombosis, pulmonary embolus, stroke, transient ischemic attack (which is often an early sign of a future stroke), and after surgery that increases the risk for blood clots.

Regular visits to the anticoagulation clinic are important for monitoring INR in patients taking warfarin. The anticoagulation team includes a doctor, nurse, pharmacist and lab members. The anticoagulation team should be informed if a patient is sick with fever, infection, diarrhea or vomiting, which can affect INR levels. Medications, foods, herbs, vitamins and alcohol can interact with anticoagulation medicine. The team may recommend that the patient avoid contact sports and exercise that can carry an increased risk of falling and injury.

If patients plan to become pregnant or are pregnant, they must contact their anticoagulation team right away because warfarin can cause serious risks to the fetus, especially during the first trimester.

\section{Clinical Pearls}

DOACs can inactivate both circulating and clot-bound activated coagulation factors. They do not require frequent monitoring because there is less variation in their efficacy for a given dose. While the overall risk of bleeding is comparable to that with warfarin, DOACs have a lower risk of intracranial bleeding. However, DOACs are expensive, and compliance is more difficult to monitor than with warfarin.

DOACs are contraindicated in patients with severe renal insufficiency, pregnancy, or prosthetic heart valves. These agents generally are not given to individuals with a body mass index (BMI) $>40 \mathrm{~kg} / \mathrm{m} 2$ or weight $>120 \mathrm{~kg}$.

DOACs are generally administered at fixed doses without laboratory monitoring, however similar laboratory testing as with warfarin must be done before administration of these agents.

Interruption of anticoagulation temporarily increases the thromboembolic risk, and continuing anticoagulation increases the risk of bleeding; both scenarios adversely affect the patient's overall health. Eye care providers must work closely with primary care providers and/or the anticoagulation team to decide upon the best course of action on an individual-patient basis for each instance of ocular bleeding. $\bullet$

CORRESPONDENCE

Len V. Koh OD PhD FAAO

Staff Optometrist

Mann-Granstaff VA Medical Center

Spokane, WA 99208

len.koh@va.gov

cel: 509-434-7032

fax: 509-434-7132 


\section{REFERENCES}

1. Goldhaber SZ, Bounameaux H. Pulmonary embolism and deep vein thrombosis. Lancet 2012;379: 1835-46.

2. Zimmerman LE. Embolism of central retinal artery: secondary to myocardial infarction with mural thrombosis. Arch Ophthalmol 1965;73:822-6.

3. Wendelboe AM, Raskob GE. Global burden of thrombosis: epidemiologic aspects. Circ Res 2016;118:1340-7.

4. Davie EW, Fujikawa K, Kisiel W. The coagulation cascade: initiation, maintenance, and regulation. Biochemistry 1991;30(43):10363-70.

5. Hoffman M, Monroe DM. Impact of non-vitamin K antagonist oral anticoagulants from a basic science perspective. Arterioscler Thromb Vasc Biol 2017;37:1812-8.

6. Bickman JK, Baglin T, Meijers JCM, Renne T. Novel targets for anticoagulants lacking bleeding risk. Curr Opin Hematol 2017;24:419-26.

7. Sozeri Y and Salim S. Anticlotting agents and the surgical management of glaucoma Curr Opin Ophthalmol 2018;29:185-9.

8. Hirsh J. Oral anticoagulants: mechanism of action, clinical effectiveness, and optimal therapeutic range. Chest 2001;119(1):8S-21S.

9. ICSH/ICTH recommendations for reporting prothrombin time in oral anticoagulant control. International Committee for Standardization in Haematology and International Committee on Thrombosis and Haemostasis. J Clin Pathol 1985;38:133-4.

10. Wells PS, Holbrook AM, Crowther RR, Hirsh J. Interactions of warfarin with drugs and food. Ann Intern Med 1994;121(9):676-83.

11. Barnes GD, Ageno W, Ansell J, Kaatz S. Recommendation on the nomenclature for oral anticoagulants: communication from the SSC of the ISTH. J Thromb Haemost 2015;13(6):1154-6.

12. Hinojar R, Jimerez-Natcher JJ, Fernandez-Golfin C, Zamorano JL. New oral anticoagulants: a practical guide for physicians. Eur Heart J Cardiovasc Pharmacother 2015;1:134-45.
13. Monaco L, Biagi C, Conti V, et al. Safety profile of DOACs. Br J Clin Pharmacol 2017;83:1532-43.

14. Sun MT, Wood MK, Chan W, et al. Risk of intraocular bleeding with novel oral anticoagulants compared with warfarin- a systemic review and meta-analysis. JAMA Ophthalmol 2017;135(8):864-87.

15. Tilanus MA, VaandragerW, Cuypers MH, Verbeek AM, Hoyng CB. Relationship between anticoagulant medication and massive intraocular hemorrhage in age-related macular degeneration. Graefes Arch Clin Exp Ophthalmol 2000;238(6):482-5.

16. Douketis JD, Berger PB, Dunn AS, et al; American College of Chest Physicians. The perioperative management of antithrombotic therapy: American College of Chest Physicians Evidence-Based Clinical Practice Guidelines (8th Edition). Chest 2008;133(6 suppl):299S339S.

17. Talany G, Guo M, Etminan M. Risk of intraocular hemorrhage with new oral anticoagulants. Eye 2017;31:628-31.

18. Kong KL, Khan J. Ophthalmic patients on antithrombotic drugs: a review and guide to perioperative management. Br J Ophthalmol 2015;99(8):1025-30.

19. Healey JS, Eikelboom J, Douketis J, et al; RE-LY Investigators. Periprocedural bleeding and thromboembolic events with dabigatran compared with warfarin: results from the Randomized Evaluation of Long-term Anticoagulation Therapy (RE-LY) randomized trial. Circulation 2012;126(3):343-8.

20. Esparaz ES, Sobel RK. Perioperative management of anticoagulants and antiplatelet agents in oculoplastic surgery. Curr Opin Ophthalmol 2015;26(5):422-8.

21. Patel R, Charles S, Jalil A. Antiplatelets and anticoagulants in vitreoretinal surgery, with a special emphasis on novel anticoagulants: a national survey and review. Graefes Arch Clin Exp Ophthalmol 2017;255:1275-85. 